\title{
Bayes-Nash Equilibrium of the Generalized First-Price Auction
}

\author{
Xiaohu Han ${ }^{1,2}$ and Shulin Liu ${ }^{1}$ \\ ${ }^{1}$ School of International Trade and Economics, University of International Business and Economics, Beijing 100029, China \\ ${ }^{2}$ Hebei Academy of Governance, Shijiazhuang 050031, China \\ Correspondence should be addressed to Shulin Liu; slliu@uibe.edu.cn
}

Received 9 June 2015; Revised 19 September 2015; Accepted 21 September 2015

Academic Editor: Anna M. Gil-Lafuente

Copyright (C) 2015 X. Han and S. Liu. This is an open access article distributed under the Creative Commons Attribution License, which permits unrestricted use, distribution, and reproduction in any medium, provided the original work is properly cited.

\begin{abstract}
We analyze the generalized first-price auction under incomplete information setting. Without setting a reserve price, the efficient symmetrical Bayes-Nash equilibrium is characterized and found to be increasing as the number of bidders is sufficiently large. Then, the explicit expression for the expected revenue of the search engine is found and the effect of the click rates of all the positions on the expected revenue is obtained. Finally, with setting of the reserve price, we have found the optimal reserve price and examine how the difference of the search engine's revenues with setting reserve price and without setting reserve price varies with the reserve price.
\end{abstract}

\section{Introduction}

With the increasing popularity of the Internet, sponsored search advertisements become one of important commercial advertisements and main revenue of the search engine. Practical importance of the sponsored search auctions has attracted much research interest of many researchers [1-3]. These papers are cornerstone of the generalized second-price auction conducted by the search engines to sell their sponsored search advertisements and have been extended in various important dimensions. Chen et al. [4] develop a model which treats the fraction of clicks allocated to each advertiser as a design variable. Thompson and Leyton-Brown [5] provide a computational analysis of perfect-information position auctions. Edelman and Schwarz [6] analyze the reserve price. Liu et al. [7] analyze the click-weights and show that although click-weighting is efficient, a profit-maximizing search engine would typically want to choose different weights. Rayo and Segal [8] analyze the information disclosure in position auction. However, all these works are executed under complete information setting. There are few papers which model the sponsored search auctions under incomplete information setting $[9,10]$. Gomes and Sweeney [10] analyze the BayesNash equilibrium of the generalized second-price auction.

The advertisement positions are sold mostly through the generalized second-price auction (GSP) by the search engine such as Google, Yahoo!, and MSN. Some search engines like Overture sell the positions through the generalized first-price auction (GFP). The selling process is as follows. An Internet user performs a query using a search engine which shows a set of search results. These results show the links the search engine has deemed relevant to the search but it also includes a list of sponsored links (paid advertisements). Each time the user clicks on a sponsored link the advertiser pays the search engine. In GSP auctions, bidders submit simultaneous bids to the seller. The highest bidder wins and pays the value of his bid. In GSP auctions, bidders submit simultaneous bids to the sellers. Again, the highest bidder wins but in this case, he pays the value of the second-highest bid.

To our best knowledge, these are a few research papers which pay attention to GFP. Szymanski and Lee [11] discuss how advertisers, by considering minimum return on investment (ROI), change their bidding and consequently the search engines revenue in sponsored search advertisement auctions for GFP, GSP, and VCG. Hoy et al. [12] study repeated GFP auctions and show that they offer powerful performance guarantees. This paper investigates GFP auctions under incomplete information under two aspects: no reserve price and setting the reserve price. We characterized the equilibrium bidding strategy and analyze its properties and examine the expected revenue of the search engine and the optimal reserve price. 
The remainder of this paper is organized as follows. In Section 2, the basic assumptions and notations are presented. Section 3 characterizes the equilibrium bidding strategy with its properties being analyzed, examines the expected revenue of the search engines, and investigates the optimal reserve price. Section 4 concludes the paper.

\section{Preliminary Assumptions and Notations}

In this section, we introduce some notations. We model GFP auction by the following assumptions:

(A1) We consider a single round static game in incomplete information setting.

(A2) There are $k$ positions and $n$ bidders with $k \leq n$.

(A3) Bidders and the search engine are risk neutral. That is, both want to maximize their expected profits.

(A4) The bidders are symmetric. That is, the valuations of all the bidders are independently and identically distributed.

(A5) Let $v_{i}$ denote the private valuation of the per click for bidder $i$. We assume that $v_{i}$ is independently drawn from the distribution $F$ on $[0,1]$ (since the valuations are not negative and infinite, we can use the interval of $[0,1]$ without loss of generalities) with the continuous positive density function $f$. The valuation of per click for bidder $i, v_{i}$, is identical across all the positions.

(A6) Let $Y_{k}^{n}$ denote the $k$ th highest of $n$ independent draws from $F$ with $G_{k: n}(\cdot)$ and $g_{k: n}(\cdot)$ denoting the distribution and density functions, respectively.

(A7) Let $\alpha_{j}$ denote the click-through rates of position $j$ for $j=1,2, \ldots, k$, satisfying $\alpha_{1} \geq \alpha_{2} \geq \cdots \geq \alpha_{k}$. The click-through rates are the same across bidders. Namely, it is related to only positions.

(A8) Let $b_{-i}^{j}$ denote the $j$ th highest bid excluding bidder $i$.

(A9) Let $C_{n}^{j}=n ! /(n-j) ! j$ ! denote the number of permutations of $j$ objects from a set of $n$.

(A10) The above assumptions are common knowledge for all bidders.

\section{Main Results}

3.1. The Equilibrium Bidding Strategy and Its Property. In setting of complete information where all information including all the valuations is common knowledge, bidders can get their desired positions. In the setting of incomplete information, since the valuations of bidders are private information, each bidder maximizes his total expected profit by treating all other bidders' valuations as private information (random variables) in order to obtain his bidding strategy.
Proposition 1 (equilibrium bidding strategy). The efficient symmetric Bayes-Nash equilibrium bidding strategy of bidder $i$ with the valuations of $v_{i}$ is given by

$$
b^{*}\left(v_{i}\right)=v_{i}-\frac{\sum_{j=1}^{k}\left(\alpha_{j}-\alpha_{j+1}\right) \int_{0}^{v_{i}} G_{j: n-1}(t) \mathrm{d} t}{\sum_{j=1}^{k}\left(\alpha_{j}-\alpha_{j+1}\right) G_{j: n-1}\left(v_{i}\right)} .
$$

(If there is only one advertising position, the equilibrium bid strategy degenerates to the bid strategy of single good. From $\alpha_{1}=1, \alpha_{j}=0$ for all $j=2,3, \ldots, k$, one has $b^{*}\left(v_{i}\right)=$ $\left.v_{i}-\int_{0}^{v_{i}} G_{1: n-1}(t) \mathrm{d} t / G_{1: n-1}\left(v_{i}\right).\right)$

Proof. Suppose that all but bidder $i$ follow the symmetric equilibrium bidding strategy $b^{*}(v)$, we will argue that it is also optimal for bidder $i$ to follow $b^{*}(v)$. We assume that equilibrium bidding strategy $b^{*}(v)$ is strictly increasing in valuation $v$. The welfare-maximizing (efficient) allocation assigns the bidder with $j$-highest valuation per click to the $j$ th highest position. Therefore, in an efficient allocation, bidder $i$ with valuation $v_{i}$ obtains the $j$ th highest position with probability of

$$
\begin{aligned}
\operatorname{Pr} & \left(b_{-i}^{j-1}>b_{i}>b_{-i}^{j}\right) \\
& =\operatorname{Pr}\left(b\left(Y_{j-1}^{n-1}\right)>b\left(v_{i}\right)>b\left(Y_{j}^{n-1}\right)\right) \\
& =\operatorname{Pr}\left(Y_{j-1}^{n-1}>v_{i}>Y_{j}^{n-1}\right) \\
& =C_{n-1}^{j-1}\left(1-F\left(v_{i}\right)\right)^{j-1} F^{n-j}\left(v_{i}\right) \\
& =G_{j: n-1}\left(v_{i}\right)-G_{j-1: n-1}\left(v_{i}\right) .
\end{aligned}
$$

If bidder $i$ with the valuation of $v_{i}$ bids $b\left(z_{i}\right)$, then he receives the expected payoff of

$$
\begin{aligned}
& \pi\left(v_{i}, z_{i}\right)=\alpha_{1} \operatorname{Pr}\left\{b\left(z_{i}\right)>b_{-i}^{1}\right\}\left(v_{i}-b\left(z_{i}\right)\right) \\
& +\alpha_{2} \operatorname{Pr}\left\{b_{-i}^{1}>b\left(z_{i}\right)>b_{-i}^{2}\right\}\left(v_{i}-b\left(z_{i}\right)\right)+\cdots \\
& +\alpha_{k} \operatorname{Pr}\left\{b_{-i}^{k-1}>b\left(z_{i}\right)>b_{-i}^{k}\right\}\left(v_{i}-b\left(z_{i}\right)\right) \\
& \quad+\alpha_{1} \operatorname{Pr}\left\{b\left(z_{i}\right)>b\left(Y_{1}^{n-1}\right)\right\}\left(v_{i}-b\left(z_{i}\right)\right) \\
& +\alpha_{2} \operatorname{Pr}\left\{b\left(Y_{1}^{n-1}\right)>b\left(z_{i}\right)>b\left(Y_{2}^{n-1}\right)\right\}\left(v_{i}-b\left(z_{i}\right)\right) \\
& +\cdots \\
& +\alpha_{k} \operatorname{Pr}\left\{b\left(Y_{k-1}^{n-1}\right)>b\left(z_{i}\right)>b\left(Y_{k}^{n-1}\right)\right\}\left(v_{i}-b\left(z_{i}\right)\right)
\end{aligned}
$$

After simplification, we have

$$
\begin{aligned}
\pi & \left(v_{i}, z_{i}\right)=\alpha_{1} G_{1: n-1}\left(z_{i}\right)\left(v_{i}-b\left(z_{i}\right)\right)+\alpha_{2}\left(G_{2: n-1}\left(z_{i}\right)\right. \\
& \left.-G_{1: n-1}\left(z_{i}\right)\right)\left(v_{i}-b\left(z_{i}\right)\right)+\cdots+\alpha_{k}\left(G_{k: n-1}\left(z_{i}\right)\right. \\
& \left.-G_{k-1: n-1}\left(z_{i}\right)\right)\left(v_{i}-b\left(z_{i}\right)\right)=\left\{G_{1: n-1}\left(z_{i}\right)\left(\alpha_{1}-\alpha_{2}\right)\right. \\
& +G_{2: n-1}\left(z_{i}\right)\left(z_{2}-z_{3}\right)+\cdots \\
& +G_{k-1: n-1}\left(z_{i}\right)\left(\alpha_{k-1}-\alpha_{k}\right)
\end{aligned}
$$




$$
\begin{aligned}
& \left.+G_{k: n-1}\left(z_{i}\right)\left(\alpha_{k}-\alpha_{k+1}\right)\right\}\left(v_{i}-b\left(z_{i}\right)\right) \\
& =\sum_{j=1}^{k}\left(\alpha_{j}-\alpha_{j+1}\right) G_{j: n-1}\left(z_{i}\right)\left(v_{i}-b\left(z_{i}\right)\right),
\end{aligned}
$$

where $\alpha_{k+1}=0$.

To achieve the goal of maximizing the expected payoff, the following necessary condition must be satisfied:

$$
\begin{aligned}
& \left.\frac{\partial \pi\left(v_{i}, z_{i}\right)}{\partial z_{i}}\right|_{z_{i}=v_{i}} \\
& =\sum_{j=1}^{k}\left(\alpha_{j}-\alpha_{j+1}\right) g_{j: n-1}\left(v_{i}\right)\left(v_{i}-b\left(v_{i}\right)\right) \\
& \quad-\sum_{j=1}^{k}\left(\alpha_{j}-\alpha_{j+1}\right) G_{j: n-1}\left(v_{i}\right) b^{\prime}\left(v_{i}\right)=0 .
\end{aligned}
$$

Simplifying yields

$$
\begin{aligned}
& \sum_{j=1}^{k}\left(\alpha_{j}-\alpha_{j+1}\right) g_{j: n-1}\left(v_{i}\right) v_{i} \\
& -\sum_{j=1}^{k}\left(\alpha_{j}-\alpha_{j+1}\right) g_{j: n-1}\left(v_{i}\right) b\left(v_{i}\right) \\
& -\sum_{j=1}^{k}\left(\alpha_{j}-\alpha_{j+1}\right) G_{j: n-1}\left(v_{i}\right) b^{\prime}\left(v_{i}\right)=0 .
\end{aligned}
$$

Furthermore, we have

$$
\begin{gathered}
\left\{\sum_{j=1}^{k}\left(\alpha_{j}-\alpha_{j+1}\right) G_{j: n-1}\left(v_{i}\right) b\left(v_{i}\right)\right\}^{\prime} \\
=\sum_{j=1}^{k}\left(\alpha_{j}-\alpha_{j+1}\right) g_{j: n-1}\left(v_{i}\right) v_{i} .
\end{gathered}
$$

Taking integral on both sides of (7) from 0 to $v_{i}$, noting that $b(0)=0$, we have

$$
\begin{aligned}
& \sum_{j=1}^{k}\left(\alpha_{j}-\alpha_{j+1}\right) G_{j: n-1}\left(v_{i}\right) b\left(v_{i}\right) \\
& =\underbrace{\int_{0}^{v_{i}} \sum_{j=1}^{k}\left(\alpha_{j}-\alpha_{j+1}\right) t g_{j: n-1}(t) \mathrm{d} t}_{\text {integration by parts }} \\
& =\sum_{j=1}^{\sum_{j}^{k}\left(\alpha_{j}-\alpha_{j+1}\right) \int_{0}^{v_{i}} t \mathrm{~d} G_{j: n-1}(t)} \\
& =\sum_{j=1}^{k}\left\{\left(\alpha_{j}-\alpha_{j+1}\right)\left(\left.t G_{j: n-1}(t)\right|_{0} ^{v_{i}}-\int_{0}^{v_{i}} G_{j: n-1}(t) \mathrm{d} t\right)\right\}
\end{aligned}
$$

$$
\begin{aligned}
= & \sum_{j=1}^{k}\left(\alpha_{j}-\alpha_{j+1}\right) G_{j: n-1}\left(v_{i}\right) v_{i} \\
& -\sum_{j=1}^{k}\left(\alpha_{j}-\alpha_{j+1}\right) \int_{0}^{v_{i}} G_{j: n-1}(t) \mathrm{d} t .
\end{aligned}
$$

Thus,

$$
b^{*}\left(v_{i}\right)=v_{i}-\frac{\sum_{j=1}^{k}\left(\alpha_{j}-\alpha_{j+1}\right) \int_{0}^{v_{i}} G_{j: n-1}(t) \mathrm{d} t}{\sum_{j=1}^{k}\left(\alpha_{j}-\alpha_{j+1}\right) G_{j: n-1}\left(v_{i}\right)} .
$$

Now, (5) is merely the necessary condition for $b^{*}\left(v_{i}\right)$ to maximize $\pi\left(v_{i}, z_{i}\right)$, but we claim that it is also sufficient, given that other bidders follow $b^{*}(\cdot)$. In fact, by (1) we have

$$
\begin{aligned}
& \left(b^{*}\left(z_{i}\right)\right)^{\prime} \\
& =\frac{\sum_{j=1}^{k}\left(\alpha_{j}-\alpha_{j+1}\right) g_{j: n-1}\left(z_{i}\right) \cdot \sum_{j=1}^{k}\left(\alpha_{j}-\alpha_{j+1}\right) \int_{0}^{z_{i}} G_{j: n-1}(t) \mathrm{d} t}{\left(\sum_{j=1}^{k}\left(\alpha_{j}-\alpha_{j+1}\right) G_{j: n-1}\left(z_{i}\right)\right)^{2}}, \\
& v_{i}-b^{*}\left(z_{i}\right)=v_{i}-z_{i}+\frac{\sum_{j=1}^{k}\left(\alpha_{j}-\alpha_{j+1}\right) \int_{0}^{z_{i}} G_{j: n-1}(t) \mathrm{d} t}{\sum_{j=1}^{k}\left(\alpha_{j}-\alpha_{j+1}\right) G_{j: n-1}\left(z_{i}\right)} .
\end{aligned}
$$

Substituting (10) and (11) into the left hand side of (5) yields

$$
\frac{\partial \pi\left(v_{i}, z_{i}\right)}{\partial z_{i}}=\sum_{j=1}^{k}\left(\alpha_{j}-\alpha_{j+1}\right) g_{j: n-1}\left(z_{i}\right)\left(v_{i}-z_{i}\right) .
$$

Because $\partial \pi\left(v_{i}, z_{i}\right) / \partial z_{i}>0$ if $z_{i}<v_{i}$ and $\partial \pi\left(v_{i}, z_{i}\right) / \partial z_{i}<0$ if $z_{i}>v_{i}$, the previous claim is true. Additionally, by (10) we have $\left(b^{*}\left(z_{i}\right)\right)^{\prime}>0$. Thus, $b(\cdot)$ is strictly increasing in valuation. The proof of Proposition 1 is completed.

Proposition 1 shows that each bidder's equilibrium bid is strictly less than his own valuation and then his expected profit will be positive in equilibrium. This is in accordance with intuition. The difference between the valuation and the equilibrium bid is called bid shading (bid shading describes the practice of a bidder placing a bid that is below what they believe a good is worth) which is strictly more than zero and denoted by sh shown as follows:

$$
\operatorname{sh}=\frac{\sum_{j=1}^{k}\left(\alpha_{j}-\alpha_{j+1}\right) \int_{0}^{v} G_{j: n-1}(t) d t}{\sum_{j=1}^{k}\left(\alpha_{j}-\alpha_{j+1}\right) G_{j: n-1}(v)} .
$$

Next we analyze how the number of bidders $n$ will affect each bidder's equilibrium bid and the bid shading.

Proposition 2. As the number of bidders $n$ becomes more and more large, each bidder's bid shading will be weakened and his equilibrium bid is more and more close to his own valuation. 
Proof. First, we rewrite (13) as

$$
\begin{aligned}
\operatorname{sh} & =\frac{\sum_{j=1}^{k} \alpha_{j} \int_{0}^{v}\left[G_{j: n-1}(t)-G_{j-1: n-1}(t)\right] \mathrm{d} t}{\sum_{j=1}^{k} \alpha_{j}\left[G_{j: n-1}(v)-G_{j-1: n-1}(v)\right]} \\
& =\frac{\sum_{j=1}^{k} \alpha_{j} C_{n-1}^{j-1} \int_{0}^{v}[1-F(t)]^{j-1} F^{n-j}(t) \mathrm{d} t}{\sum_{j=1}^{k} \alpha_{j} C_{n-1}^{j-1}[1-F(v)]^{j-1} F^{n-j}(v)} .
\end{aligned}
$$

Because of $\max _{t}\left(a_{t} / b_{t}\right) \geq \sum_{t} a_{t} / \sum_{t} b_{t} \geq \min _{t}\left(a_{t} / b_{t}\right)$, we have

$$
\begin{gathered}
\max _{j} \frac{\alpha_{j} C_{n-1}^{j-1} \int_{0}^{v}[1-F(t)]^{j-1} F^{n-j}(t) \mathrm{d} t}{\alpha_{j} C_{n-1}^{j-1}[1-F(v)]^{j-1} F^{n-j}(v)} \geq \mathrm{sh} \\
\geq \min _{j} \frac{\alpha_{j} C_{n-1}^{j-1} \int_{0}^{v}[1-F(t)]^{j-1} F^{n-j}(t) \mathrm{d} t}{\alpha_{j} C_{n-1}^{j-1}[1-F(v)]^{j-1} F^{n-j}(v)} .
\end{gathered}
$$

Based on the mean value theorem of integrals, we have

$$
\begin{array}{r}
\max _{j} \frac{[1-F(\xi)]^{j-1} F^{n-j}(\xi) v}{[1-F(v)]^{j-1} F^{n-j}(v)} \geq s h \\
\geq \min _{j} \frac{[1-F(\xi)]^{j-1} F^{n-j}(\xi) v}{[1-F(v)]^{j-1} F^{n-j}(v)},
\end{array}
$$

where $\xi \in(0, v)$. Since $F(\xi)<F(v)$, limits of two sides of (16) and sh will be zero as the number of bidders $n$ approaches to infinity. Thus, the limit of each bidder's equilibrium bid is his own valuation by (1) as the number of bidders $n$ approaches to infinity. This competes the proof of Proposition 2.

Proposition 2 is consistent with intuition. This is because of the fact that the more the number of bidders is, the more competitive the bidding is.

3.2. The Equilibrium Revenue and Properties. After we get the expression of the equilibrium bidding strategy, we can find the expected revenue of the search engine paid by the bidders and make comparative static analysis of it.

Proposition 3 (expected equilibrium revenue). The expected equilibrium revenue of the search engine is given by

$$
\begin{aligned}
E & \left(R_{\text {total }}\right) \\
& =n \sum_{j=1}^{k}\left(\alpha_{j}-\alpha_{j+1}\right) \int_{0}^{1} t(1-F(t)) \mathrm{d} G_{j: n-1}(t) .
\end{aligned}
$$

Proof. Using the expected payment of a bidder, we can compute the search engine's expected revenue. Notice that the expected number of clicks for bidder $i$ with the valuation of $v_{i}$ is

$$
\begin{aligned}
\overline{\alpha\left(v_{i}\right)}= & G_{1: n-1}\left(v_{i}\right) \alpha_{1}+\left[G_{2: n-1}\left(v_{i}\right)-G_{1: n-1}\left(v_{i}\right)\right] \alpha_{2} \\
& +\cdots+\left[G_{k: n-1}\left(v_{i}\right)-G_{k-1: n-1}\left(v_{i}\right)\right] \alpha_{k} \\
= & \sum_{j=1}^{k} G_{j: n-1}\left(v_{i}\right)\left(\alpha_{j}-\alpha_{j+1}\right),
\end{aligned}
$$

and the expected payment of bidder $i$ is

$$
\begin{aligned}
\overline{\alpha\left(v_{i}\right)} b^{*}\left(v_{i}\right)= & v_{i} \sum_{j=1}^{k} G_{j: n-1}\left(v_{i}\right)\left(\alpha_{j}-\alpha_{j+1}\right) \\
& -\sum_{j=1}^{k}\left(\alpha_{j}-\alpha_{j+1}\right) \int_{0}^{v_{i}} G_{j: n-1}(t) \mathrm{d} t .
\end{aligned}
$$

Since the valuation $v_{i}$ is a private information of bidder $i$ and is independently drawn from the density function $f(\cdot)$, the expected revenue of the search engine paid by bidder $i$ is

$$
\begin{aligned}
E(R)= & \int_{0}^{1} \overline{\alpha(v)} b^{*}(v) f(v) \mathrm{d} v \\
= & \int_{0}^{1} v \sum_{j=1}^{k} G_{j: n-1}(v)\left(\alpha_{j}-\alpha_{j+1}\right) f(v) \mathrm{d} v \\
& -\int_{0}^{1} \sum_{j=1}^{k}\left(\alpha_{j}-\alpha_{j+1}\right) \int_{0}^{v} G_{j: n-1}(t) \mathrm{d} t f(v) \mathrm{d} v .
\end{aligned}
$$

Exchanging the order of the integral yields

$$
\begin{aligned}
& E(R)=\sum_{j=1}^{k}\left(\alpha_{j}-\alpha_{j+1}\right) \int_{0}^{1} v G_{j: n-1}(v) f(v) \mathrm{d} v \\
& -\sum_{j=1}^{k}\left(\alpha_{j}-\alpha_{j+1}\right) \underbrace{\int_{0}^{1} \int_{0}^{v} G_{j: n-1}(t) \mathrm{d} t f(v) \mathrm{d} v}_{\text {change the order of integral }} \\
& E(R)=\sum_{j=1}^{k}\left(\alpha_{j}-\alpha_{j+1}\right) \underbrace{\int_{0}^{1} v G_{j: n-1}(v) f(v) \mathrm{d} v}_{\text {integration by parts }} \\
& \quad-\sum_{j=1}^{k}\left(\alpha_{j}-\alpha_{j+1}\right) \int_{0}^{1} \int_{t}^{1} G_{j: n-1}(t) f(v) \mathrm{d} v \mathrm{~d} t \\
& \quad=\sum_{j=1}^{k}\left(\alpha_{j}-\alpha_{j+1}\right) \\
& \quad .\left\{\left.v G_{j: n-1}(v) F(v)\right|_{0} ^{1}-\int_{0}^{1} F(v) \mathrm{d}\left[v G_{j: n-1}(v)\right]\right\} \\
& \quad-\sum_{j=1}^{k}\left(\alpha_{j}-\alpha_{j+1}\right) \int_{0}^{1} G_{j: n-1}(t)[1-F(t)] \mathrm{d} t .
\end{aligned}
$$

Further simplification yields

$$
\begin{gathered}
E(R)=\sum_{j=1}^{k}\left(\alpha_{j}-\alpha_{j+1}\right)\left[1-\int_{0}^{1} F(v) v \mathrm{~d}_{j: n-1}(v)\right. \\
\left.-\int_{0}^{1} F(v) G_{j: n-1}(v) \mathrm{d} v\right]-\sum_{j=1}^{k}\left(\alpha_{j}-\alpha_{j+1}\right)
\end{gathered}
$$




$$
\begin{aligned}
& \int_{0}^{1} G_{j: n-1}(t)[1-F(t)] \mathrm{d} t=\sum_{j=1}^{k}\left(\alpha_{j}-\alpha_{j+1}\right)[1 \\
& -\int_{0}^{1} F(v) v \mathrm{~d} G_{j: n-1}(v)-\underbrace{1}_{0} G_{j: n-1}(t) \mathrm{d} t \\
& =\sum_{j=1}^{k}\left(\alpha_{j}-\alpha_{j+1}\right)\left[1-\int_{0}^{1} F(v) v \mathrm{~d} G_{j: n-1}(v)\right. \\
& \left.-\left.t G_{j: n-1}(t)\right|_{0} ^{1}+\int_{0}^{1} t \mathrm{~d} G_{j: n-1}(t)\right]=\sum_{j=1}^{k}\left(\alpha_{j}-\alpha_{j+1}\right) \\
& \cdot\left[\int_{0}^{1} t \mathrm{~d} G_{j: n-1}(t)\right. \\
& \left.-\int_{0}^{1} v F(v) \mathrm{d} G_{j: n-1}(v)\right]=\sum_{j=1}^{1}\left(\alpha_{j}-\alpha_{j+1}\right) \\
& -F(t)] \mathrm{d} G_{j: n-1}(t) .
\end{aligned}
$$

From the symmetrical characteristic of bidders, we have $E\left(R_{\text {total }}\right)=n E(R)$ as required. The proof of Proposition 3 is completed.

If there is only one advertising position, the expected equilibrium revenue degenerates to the revenue of single good. From $\alpha_{1}=1, \alpha_{j}=0$ for all $j=2,3, \ldots, k$, one has $E\left(R_{\text {total }}\right)=n \int_{0}^{1} t(1-F(t)) \mathrm{d} G_{1: n-1}(t)=n \int_{0}^{1} t(1-F(t)) \mathrm{d} F^{n-1}(t)$ $=\int_{0}^{1} t n(n-1) F^{n-2}(t)(1-F(t)) f(t) \mathrm{d} t=\int_{0}^{1} \operatorname{tg}_{2: n}(t) \mathrm{d} t=E\left[Y_{2}^{n}\right]$.

The click-through rates are vital parameters. Next we analyze their effects on the expected revenue of the search engine.

Proposition 4 (comparative statics). The expected equilibrium revenue of the search engine is

(i) increasing in the click-through rate $\alpha_{1}$ of the first position,

(ii) increasing in the click-through rate $\alpha_{j}$ of position $j$ if and only if

$$
\begin{gathered}
\int_{0}^{1} t F^{n-j-1}(t)[1-F(t)]^{j-1} f(t) \\
\cdot[n-j-(n-1) F(t)] \mathrm{d} t \geq 0 .
\end{gathered}
$$

Proof. Differentiating (17) with respect to $\alpha_{1}$ and $\alpha_{j}$ for $j \geq 2$, respectively, we have

$$
\begin{aligned}
& \frac{\partial E\left(R_{\text {total }}\right)}{\partial \alpha_{1}}=n \int_{0}^{1} t[1-F(t)] \mathrm{d} G_{1: n-1}(t)>0 \\
& \quad \text { for } j=1, \\
& \frac{\partial E\left(R_{\text {total }}\right)}{\partial \alpha_{j}}=n \int_{0}^{1} t[1 \\
& -F(t)] \mathrm{d}\left[G_{j: n-1}(t)-G_{j-1: n-1}(t)\right] \\
& =n \int_{0}^{1} t[1-F(t)] \mathrm{d}\left\{C_{n-1}^{j-1}[1-F(t)]^{j-1} F^{n-j}(t)\right\} \\
& =n C_{n-1}^{j-1} \int_{0}^{1} t F^{n-j-1}(t)[1-F(t)]^{j-1} f(t)[n-j \\
& -(n-1) F(t)] \mathrm{d} t
\end{aligned}
$$

for $j \geq 2$. Then, parts (i) and (ii) immediately come from the above two equations, respectively. The proof of Proposition 4 is completed.

Because the top position has the largest probability of being clicked among all the positions and then brings more revenues to the search engine, the more the click rate of top position, the more the revenues which the search engine receives from it. Thus, Proposition 4 is in accordance with practice.

3.3. The Optimal Reserve Price. This section considers GSP auction with a uniform reserve price $r$ set by the search engine. We assume that any bidder will lose if his bid is less than the reserve price $r$. We firstly find the equilibrium bidding strategy. Secondly, we find the expected revenue of the search engine. Finally, we maximize the expected revenue to find the optimal reserve price. To do these, we need quasiefficient allocation.

Definition 5. An allocation of positions to bidders is quasiefficient allocation, if the following conditions are satisfied $[10]$.

(i) The bidders with higher value are assigned higher positions.

(ii) No position is left vacant when there is a bidder with valuation $v \geq r$ who is not assigned any position.

We start our analysis of GFP with reserve price $r$ by its unique quasi-efficient Bayes-Nash equilibrium. However, the reserve price is always set by search engine to the extent that it influences bidding behavior. Similar to the process of solving equilibrium bid strategy without reserve price, we can obtain the one with reserve price.

Proposition 6 (equilibrium bidding strategy with the reserve price). With the existence of the uniform reserve price $r \geq 0$, 
the quasi-efficient Bayes-Nash equilibrium bidding strategy is given by

$$
b_{r}^{*}\left(v_{i}\right)=v_{i}-\frac{\sum_{j=1}^{k}\left(\alpha_{j}-\alpha_{j+1}\right) \int_{r}^{v_{i}} G_{j: n-1}(t) \mathrm{d} t}{\sum_{j=1}^{k}\left(\alpha_{j}-\alpha_{j+1}\right) G_{j: n-1}\left(v_{i}\right)} .
$$

Proof. See Appendix.

If there is only one advertising position, the equilibrium bid strategy degenerates to the bid strategy of single good. From $\alpha_{1}=1, \alpha_{j}=0$ for all $j=2,3, \ldots, k$, one has $b^{*}\left(v_{i}\right)=$ $v_{i}-\int_{r}^{v_{i}} G_{1: n-1}(t) \mathrm{d} t / G_{1: n-1}\left(v_{i}\right)$.

Proposition 6 has similar interpretation and intuition with Proposition 1.

Proposition 7 (expect equilibrium revenue with the reserve price). With the existence of the uniform reserve price $r$, the expected equilibrium revenue of the search engine is

$$
\begin{gathered}
E\left(R_{\text {rtotal }}\right)=n \sum_{j=1}^{k}\left(\alpha_{j}-\alpha_{j+1}\right) \\
\cdot\left\{\int_{r}^{1} t(1-F(t)) \mathrm{d} G_{j: n-1}(t)\right. \\
\left.+G_{j: n-1}(r) r(1-F(r))\right\} .
\end{gathered}
$$

Proof. See Appendix.

For the search engine, setting the uniform reserve price is double-edged sword. That is, smaller reserve price decreases the probability of the inefficient event in which no sale occurs; larger reserve price eliminates more bidders and then reduces the competition of bidders. Next, we will probe the optimal reserve price. Remarkably, the optimal reserve price depends neither on the number of bidders nor on the number of available positions.

Proposition 8 (the optimal reserve price). If the distribution $F(v)$ satisfies the increasing hazard rate condition, the optimal reserve price ${ }^{*}$ is determined by

$$
r^{*}=\frac{1-F\left(r^{*}\right)}{f\left(r^{*}\right)} \quad \text { for } k=1,2, \ldots, K .
$$

Proof. Differentiating (25) with respect to $r$ leads to

$$
\begin{aligned}
& \frac{\partial E\left(R_{r \text { total }}\right)}{\partial r} \\
& \quad=n \sum_{j=1}^{k}\left(\alpha_{j}-\alpha_{j+1}\right) G_{j: n-1}(r)(1-F(r)-r f(r)) \\
& \quad=0
\end{aligned}
$$

and then we have $1-F(r)-r f(r)=0$, and when the distribution $F(v)$ satisfies the increasing hazard rate condition, there must be $r^{*}$ satisfying $r^{*}=\left(1-F\left(r^{*}\right)\right) / f\left(r^{*}\right)$.
Proposition 8 suggests that the search engine should set the reserve price satisfying (27) in order to maximize his expected revenue.

Proposition 9 (comparative statics). The difference between the search engine's revenues under setting the reserve price $r$ and no reserve price is increasing in $r$ if $r<r^{*}$ and decreasing in $r$ if $r>r^{*}$. In particular, the difference achieves a maximum at the optimal reserve price $r^{*}$.

Proof. From (17) we have

$$
\begin{aligned}
& E\left(R_{\text {total }}\right)=n \sum_{j=1}^{k}\left(\alpha_{j}-\alpha_{j+1}\right)\{\underbrace{\int_{0}^{r} t(1-F(t)) \mathrm{d} G_{j: n-1}(t)}_{\text {integration by parts }} \\
& \left.+\int_{r}^{1} t(1-F(t)) \mathrm{d} G_{j: n-1}(t)\right\}=n \sum_{j=1}^{k}\left(\alpha_{j}-\alpha_{j+1}\right) \\
& +\left\{r(1-F(r)) G_{j: n-1}(r)\right. \\
& +\int_{0}^{r} G_{j: n-1} \mathrm{~d} t(1-F(t)) \\
& \left.+\int_{r}^{1} t(1-F(t)) \mathrm{d} G_{j: n-1}(t)\right\}=n \sum_{j=1}^{k}\left(\alpha_{j}-\alpha_{j+1}\right) \\
& +\int_{0}^{r} G_{j: n-1}(t) \mathrm{d} t(1-F(t))=E\left(R_{r t o t a l}\right) \\
& \left.+\int_{r=1}^{1} t(1-F(t)) \mathrm{d} G_{j: n-1}(t)\right\}-n \sum_{j=1}^{k}\left(\alpha_{j}-\alpha_{j+1}\right) \\
& \quad+r(1-F(r)) G_{j: n-1}(r)
\end{aligned}
$$

Thus, $E\left(R_{r \text { total }}\right)-E\left(R_{\text {total }}\right)=\varphi(r)$, where $\varphi(r)$ is called the incremental revenue function. Different reserve price $r$ yields different incremental expected revenue $\left(\varphi(r)=E\left(R_{r \text { total }}\right)-\right.$ $\left.E\left(R_{\text {total }}\right)\right)$. Since the distribution $F(v)$ satisfies the increasing hazard rate condition, we have

$$
\begin{aligned}
& \frac{\partial \varphi(r)}{\partial r} \\
& \quad n \sum_{j=1}^{k}\left(\alpha_{j}-\alpha_{j+1}\right) G_{j: n-1}(r)(1-F(r)-r f(r)) \\
& \quad<0, \quad \text { if } r>r^{*},
\end{aligned}
$$




$$
\begin{aligned}
& \frac{\partial \varphi(r)}{\partial r} \\
& \quad=n \sum_{j=1}^{k}\left(\alpha_{j}-\alpha_{j+1}\right) G_{j: n-1}(r)(1-F(r)-r f(r)) \\
& \quad=0, \quad \text { if } r=r^{*}, \\
& \frac{\partial \varphi(r)}{\partial r} \\
& \quad=n \sum_{j=1}^{k}\left(\alpha_{j}-\alpha_{j+1}\right) G_{j: n-1}(r)(1-F(r)-r f(r)) \\
& >0, \quad \text { if } r<r^{*},
\end{aligned}
$$

as required.

Proposition 9 shows how the incremental expected revenue of the search engine varies with the reserve price in detail. It is consistent with Proposition 8.

\section{Conclusion}

The games of incomplete information are the most central to the sponsored search auction. Under incomplete information we analyze the first-price auction. We have found the explicit expression for the equilibrium bidding strategy without setting the reserve price by the search engine. We make some comparative static analyses. It is concluded that the bidder's equilibrium bidding strategy is approaching his valuation as the number of bidders is sufficiently larger. This implies that sufficiently larger number of bidders benefits the search engine. We have found that the expected revenue of the search engine is increasing in the click rate of the top position. We also give a necessary and sufficient condition under which the expected revenue of the search engine is increasing in the click rates of other positions. Given that the search engine sets the reserve price, we have found the expression for determining the optimal reserve price. We have not made comparisons between our results of GFP and that of GSP by Gomes and Sweeney [10]. This is an interesting topic left for future study.

\section{Appendix}

Proof of Proposition 6. Similar to the proof of Proposition 1, suppose that all but bidder $i$ follow the equilibrium bidding strategy $b_{r}^{*}(v)$. We will argue the case that it is also optimal for bidder $i$ to follow $b_{r}^{*}(v)$. We assume that equilibrium bidding strategy $b_{r}^{*}(v)$ is strictly increasing in valuation. The welfaremaximizing (efficient) allocation assigns the bidder with $j$ highest value per click to the $j$ th highest position.

Therefore, in an efficient equilibrium, bidder $i$ with value $v_{i} \geq r$ obtains the $j$ th highest position with probability

$$
\operatorname{Pr}\left(b_{-i}^{j-1}>b_{i}>b_{-i}^{j}\right)=G_{j: n-1}\left(v_{i}\right)-G_{j-1: n-1}\left(v_{i}\right) .
$$

Then, if bidder $i$ with the valuation of $v_{i}$ bids $b\left(z_{i}\right)$, then he will receive an expected payoff of

$$
\pi\left(v_{i}, z_{i}\right)=\sum_{j=1}^{k}\left(\alpha_{j}-\alpha_{j+1}\right) G_{j: n-1}\left(z_{i}\right)\left(v_{i}-b\left(z_{i}\right)\right),
$$

where $\alpha_{k+1}=0$.

To achieve the goal of maximizing the expected payoff, the following necessary condition must be satisfied:

$$
\begin{aligned}
& \left.\frac{\partial \pi\left(v_{i}, z_{i}\right)}{\partial z_{i}}\right|_{z_{i}=v_{i}} \\
& =\left\{\sum_{j=1}^{k}\left(\alpha_{j}-\alpha_{j+1}\right) g_{j: n-1}\left(z_{i}\right)\left(v_{i}-b\left(z_{i}\right)\right)\right. \\
& \left.-\sum_{j=1}^{k}\left(\alpha_{j}-\alpha_{j+1}\right) G_{j: n-1}\left(z_{i}\right) b^{\prime}\left(z_{i}\right)\right\}\left.\right|_{z_{i}=v_{i}}=0 .
\end{aligned}
$$

Further simplification yields

$$
\begin{gathered}
\left\{\sum_{j=1}^{k}\left(\alpha_{j}-\alpha_{j+1}\right) G_{j: n-1}\left(v_{i}\right) b\left(v_{i}\right)\right\}^{\prime} \\
=\sum_{j=1}^{k}\left(\alpha_{j}-\alpha_{j+1}\right) g_{j: n-1}\left(v_{i}\right) v_{i} .
\end{gathered}
$$

Taking integral of both sides of (A.4) from $r$ to $v_{i}$, noting that $b(r)=r$, we have

$$
\begin{aligned}
& \sum_{j=1}^{k}\left(\alpha_{j}-\alpha_{j+1}\right) G_{j: n-1}\left(v_{i}\right) b\left(v_{i}\right)-\sum_{j=1}^{k}\left(\alpha_{j}-\alpha_{j+1}\right) \\
& \cdot G_{j: n-1}(r) b(r)=\int_{r}^{v_{i}} \sum_{j=1}^{k}\left(\alpha_{j}-\alpha_{j+1}\right) t g_{j: n-1}(t) \mathrm{d} t \\
& =\sum_{j=1}^{k}\left(\alpha_{j}-\alpha_{j+1}\right) \underbrace{\int_{r}^{v_{i}} t \mathrm{~d} G_{j: n-1}(t)}_{\text {integration by parts }} \\
& =\sum_{j=1}^{k}\left\{\left(\alpha_{j}-\alpha_{j+1}\right)\right. \\
& \left.\cdot\left(\left.t G_{j: n-1}(t)\right|_{r} ^{v_{i}}-\int_{r}^{v_{i}} G_{j: n-1}(t) \mathrm{d} t\right)\right\}=\sum_{j=1}^{k}\left(\alpha_{j}\right. \\
& \left.\quad-\alpha_{j+1}\right) G_{j: n-1}\left(v_{i}\right) v_{i}-\sum_{j=1}^{k}\left(\alpha_{j}-\alpha_{j+1}\right) G_{j: n-1}(r) r \\
& \quad-\sum_{j=1}^{k}\left(\alpha_{j}-\alpha_{j+1}\right) \int_{r}^{v_{i}} G_{j: n-1}(t) \mathrm{d} t .
\end{aligned}
$$


Thus,

$$
b_{r}^{*}\left(v_{i}\right)=v_{i}-\frac{\sum_{j=1}^{k}\left(\alpha_{j}-\alpha_{j+1}\right) \int_{r}^{v_{i}} G_{j: n-1}(t) \mathrm{d} t}{\sum_{j=1}^{k}\left(\alpha_{j}-\alpha_{j+1}\right) G_{j: n-1}\left(v_{i}\right)} .
$$

Now (A.3) is merely a necessary condition for $b_{r}^{*}\left(v_{i}\right)$ to maximize $\pi\left(v_{i}, z_{i}\right)$, but we claim that it is also sufficient, given that other bidders follow $b_{r}^{*}(\cdot)$. In fact, by (26) we have

$$
\begin{aligned}
\left(b_{r}^{*}\left(z_{i}\right)\right)^{\prime} & =\frac{\sum_{j=1}^{k}\left(\alpha_{j}-\alpha_{j+1}\right) g_{j: n-1}\left(z_{i}\right) \cdot \sum_{j=1}^{k}\left(\alpha_{j}-\alpha_{j+1}\right) \int_{r}^{z_{i}} G_{j: n-1}(t) \mathrm{d} t}{\left(\sum_{j=1}^{k}\left(\alpha_{j}-\alpha_{j+1}\right) G_{j: n-1}\left(z_{i}\right)\right)^{2}}, \\
v_{i}-b_{r}^{*}\left(z_{i}\right) & =v_{i}-z_{i}+\frac{\sum_{j=1}^{k}\left(\alpha_{j}-\alpha_{j+1}\right) \int_{r}^{z_{i}} G_{j: n-1}(t) \mathrm{d} t}{\sum_{j=1}^{k}\left(\alpha_{j}-\alpha_{j+1}\right) G_{j: n-1}\left(z_{i}\right)} .
\end{aligned}
$$

Substituting (A.7) and (A.8) into the left hand side of (A.3) yields

$$
\frac{\partial \pi\left(v_{i}, z_{i}\right)}{\partial z_{i}}=\sum_{j=1}^{k}\left(\alpha_{j}-\alpha_{j+1}\right) g_{j: n-1}\left(z_{i}\right)\left(v_{i}-z_{i}\right) .
$$

Because $\partial \pi\left(v_{i}, z_{i}\right) / \partial z_{i}>0$ if $z_{i}<v_{i}$ and $\partial \pi\left(v_{i}, z_{i}\right) / \partial z_{i}<0$ if $z_{i}>v_{i}$ the sufficient condition is satisfied. Based on (A.7), $\left(b_{r}^{*}\left(z_{i}\right)\right)^{\prime}>0$. Thus, $b(\cdot)$ is strictly increasing in valuation. The proof of Proposition 6 is completed.

Proof of Proposition 7. Similar to the proof of Proposition 3, the expected payment of bidder $i$ is

$$
\begin{aligned}
\overline{\alpha\left(v_{i}\right)} b_{r}^{*}\left(v_{i}\right)= & v_{i} \sum_{j=1}^{k} G_{j: n-1}\left(v_{i}\right)\left(\alpha_{j}-\alpha_{j+1}\right) \\
& -\sum_{j=1}^{k}\left(\alpha_{j}-\alpha_{j+1}\right) \int_{r}^{v_{i}} G_{j: n-1}(t) \mathrm{d} t .
\end{aligned}
$$

For the value $v_{i} \geq r$, the expected revenue of search engine gaining from bidder $i$ is

$$
\begin{aligned}
& E\left(R_{r}\right)=\int_{r}^{1} \overline{\alpha(v)} b_{r}^{*}(v) f(v) \mathrm{d} v \\
& =\int_{r}^{1} v \sum_{j=1}^{k} G_{j: n-1}(v)\left(\alpha_{j}-\alpha_{j+1}\right) f(v) \mathrm{d} v \\
& \quad-\int_{r}^{1} \sum_{j=1}^{k}\left(\alpha_{j}-\alpha_{j+1}\right) \int_{r}^{v} G_{j: n-1}(t) \mathrm{d} t f(v) \mathrm{d} v .
\end{aligned}
$$

Exchanging the order of the integral yields

$$
\begin{array}{r}
E\left(R_{r}\right)=\sum_{j=1}^{k}\left(\alpha_{j}-\alpha_{j+1}\right) \int_{r}^{1} v G_{j: n-1}(v) \mathrm{d} F(v) \\
-\sum_{j=1}^{k}\left(\alpha_{j}-\alpha_{j+1}\right) \int_{r}^{1} \int_{t}^{1} G_{j: n-1}(t) \mathrm{d} t f(v) \mathrm{d} v \\
=\sum_{j=1}^{k}\left(\alpha_{j}-\alpha_{j+1}\right)\left\{\left.v G_{j: n-1}(v) F(v)\right|_{r} ^{1}\right.
\end{array}
$$

$$
\begin{aligned}
& \left.\quad-\int_{r}^{1} F(v) \mathrm{d}\left[v G_{j: n-1}(v)\right]\right\}-\sum_{j=1}^{k}\left(\alpha_{j}-\alpha_{j+1}\right) \\
& \quad \cdot \int_{r}^{1} G_{j: n-1}(t)(1-F(t)) \mathrm{d} t, \\
& E\left(R_{r}\right)=\sum_{j=1}^{k}\left(\alpha_{j}-\alpha_{j+1}\right)\left\{1-G_{j: n-1}(r) r F(r)\right. \\
& \left.\quad-\int_{r}^{1} F(v) v \mathrm{~d} G_{j: n-1}(v)-\int_{r}^{1} F(v) G_{j: n-1}(v) \mathrm{d} v\right\} \\
& \quad-\sum_{j=1}^{k}\left(\alpha_{j}-\alpha_{j+1}\right) \int_{r}^{1} G_{j: n-1}(t)(1-F(t)) \mathrm{d} t \\
& \left.\quad+\int_{r}^{1} t \mathrm{~d} G_{j: n-1}(t)\right\}=\sum_{j=1}^{k}\left(\alpha_{j}-\alpha_{j+1}\right) \\
& \quad-\int_{r}^{1} F(v) \mathrm{d} G_{j: n-1}(v)+G_{j: n-1}(r) r \\
& \quad=\sum_{j=1}^{k}\left(\alpha_{j}-\alpha_{j+1}\right)\left\{1-G_{j: n-1}(r) r F(r)\right. \\
& \left.\quad-\int_{r}^{1} F(v) v \mathrm{~d} G_{j: n-1}(v)-\int_{j: n-1}^{1}(t) \mathrm{d} t\right\}, \\
& \quad
\end{aligned}
$$




$$
\begin{aligned}
& \left.-G_{j: n-1}(r) r F(r)\right)=\sum_{j=1}^{k}\left(\alpha_{j}-\alpha_{j+1}\right) \\
& \cdot\left\{\int_{r}^{1} t(1-F(t)) \mathrm{d} G_{j: n-1}(t)\right. \\
& \left.+G_{j: n-1}(r) r(1-F(r))\right\} .
\end{aligned}
$$

From the symmetrical characteristic of bidders, we have

$$
\begin{aligned}
& E\left(R_{r \text { total }}\right)=n E\left(R_{r}\right)=n \sum_{j=1}^{k}\left(\alpha_{j}-\alpha_{j+1}\right) \\
& \quad \cdot\left\{\int_{r}^{1} t(1-F(t)) \mathrm{d} G_{j: n-1}(t)\right. \\
& \left.+G_{j: n-1}(r) r(1-F(r))\right\} .
\end{aligned}
$$

Proposition 7 is completed.

\section{Conflict of Interests}

The authors declare that there is no conflict of interests regarding the publication of this paper.

\section{Acknowledgment}

This work was supported by the National Science Foundation of China under Grant no. 71171052.

\section{References}

[1] H. R. Varian, "Position auctions," International Journal of Industrial Organization, vol. 25, no. 6, pp. 1163-1178, 2007.

[2] B. Edelman, M. Ostrovsky, and M. Schwarz, "Internet advertising and the generalized second-price auction," American Economic Review, vol. 97, no. 1, pp. 242-259, 2007.

[3] G. Aggarwal, A. Goel, and R. Motwani, "Truthful auctions for pricing search keywords," in Proceedings of the 7th ACM Conference on Electronic Commerce (EC '06), pp. 1-7, ACM, Ann Arbor, Mich, USA, June 2006.

[4] J. Chen, D. Liu, and A. B. Whinston, "Auctioning keywords in online search," Journal of Marketing, vol. 73, no. 4, pp. 125-141, 2009.

[5] D. R. M. Thompson and K. Leyton-Brown, "Computational analysis of perfect-information position auctions," in Proceedings of the ACM Conference on Electronic Commerce (EC '09), pp. 51-60, Stanford, Calif, USA, July 2009.

[6] B. Edelman and M. Schwarz, "Optimal auction design and equilibrium selection in sponsored search auctions," American Economic Review, vol. 100, no. 2, pp. 597-602, 2010.

[7] D. Liu, J. Chen, and A. B. Whinston, "Ex-ante information and the design of keyword auction," Information Systems Research, vol. 21, no. 1, pp. 133-153, 2010.

[8] L. Rayo and I. Segal, "Optimal information disclosure," Journal of Political Economy, vol. 118, no. 5, pp. 949-987, 2010.
[9] R. P. Leme and E. Tardos, "Pure and Bayes-Nash price of anarchy for the generalized second-price auction," in Proceedings of the 1st Annual IEEE Symposium on Foundations of Computer Science (FOCS '10), pp. 735-744, IEEE, Las Vegas, Nev, USA, 2010.

[10] R. Gomes and K. Sweeney, "Bayes-Nash equilibria of the generalized second-price auction," Games and Economic Behavior, vol. 86, pp. 421-437, 2014.

[11] B. K. Szymanski and J. Lee, "Impact of ROI on bidding and revenue in sponsored search advertise-ment auctions," in Proceedings of the 2nd Workshop on Sponsored Search Auctions, Ann Arbor, Mich, USA, 2006.

[12] D. Hoy, K. Jain, and C. A. Wilkens, "A dynamic axiomatic approach to first-price auctions," in Proceedings of the 14th ACM Conference on Electronic Commerce (EC '13), pp. 583-584, ACM, Philadelphia, Pa, USA, June 2013. 


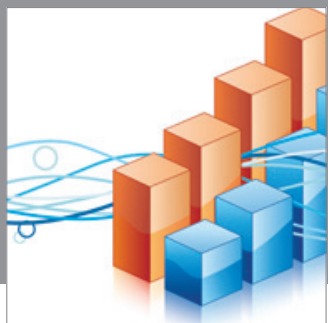

Advances in

Operations Research

mansans

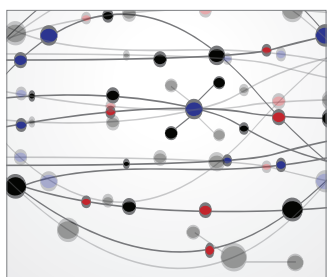

The Scientific World Journal
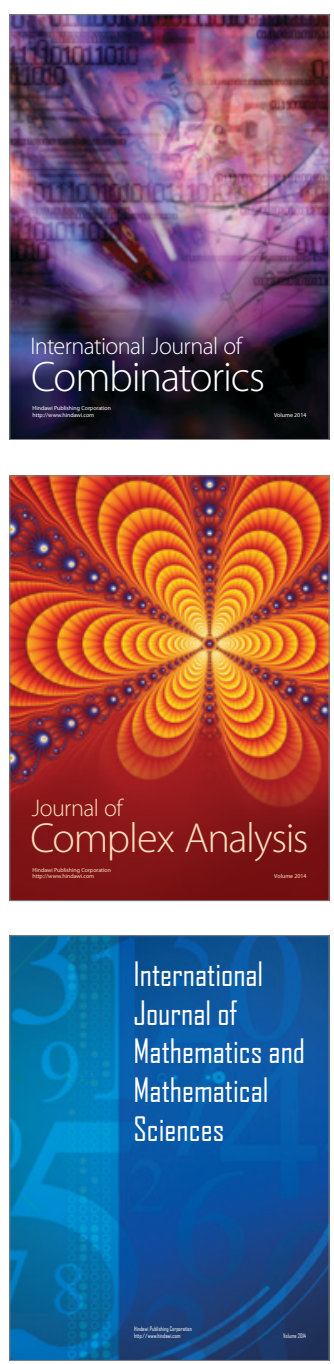
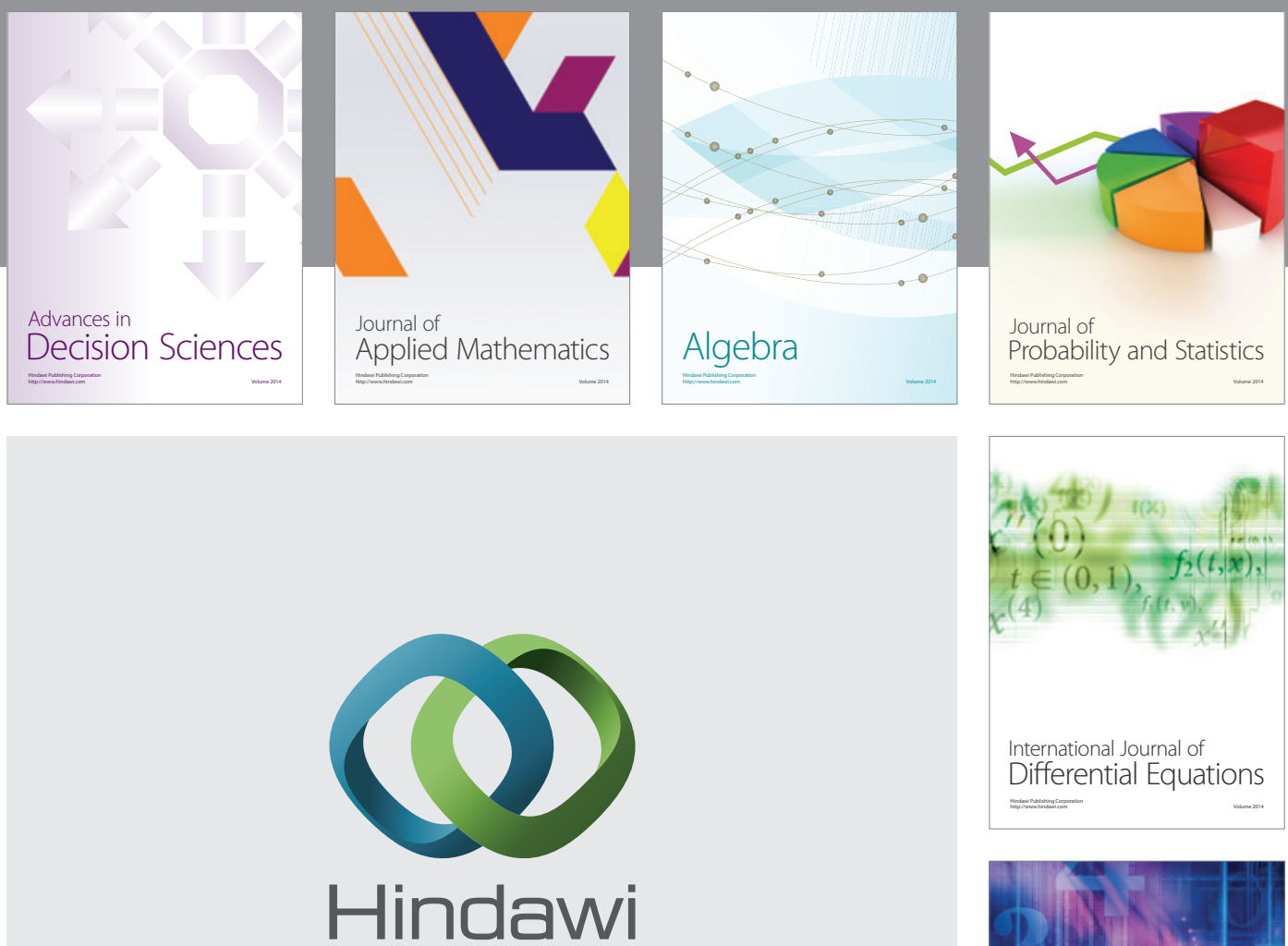

Submit your manuscripts at http://www.hindawi.com
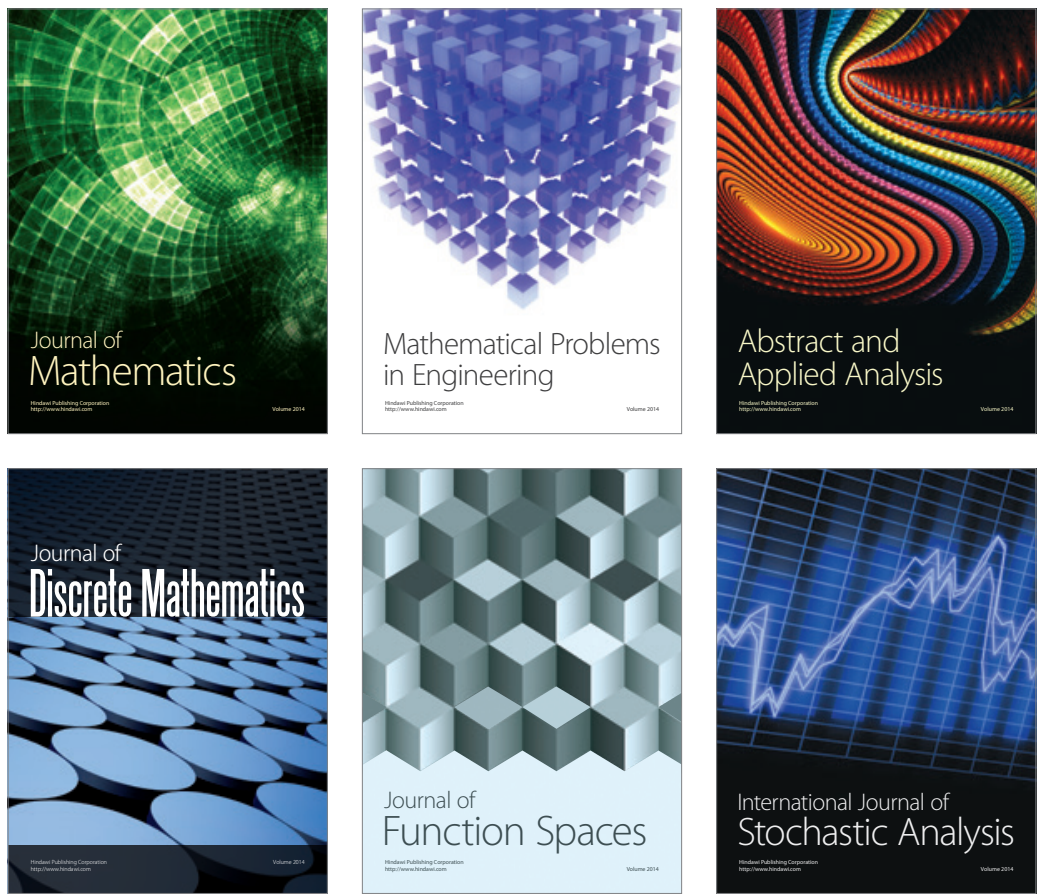

Journal of

Function Spaces

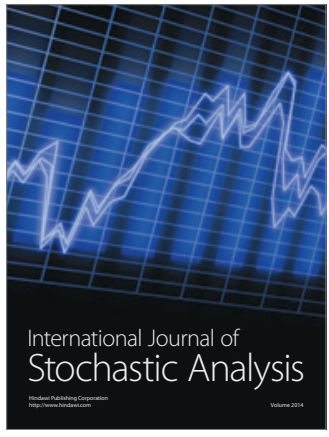

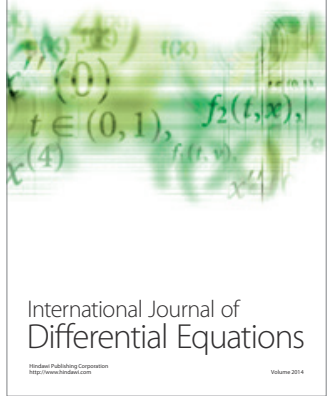
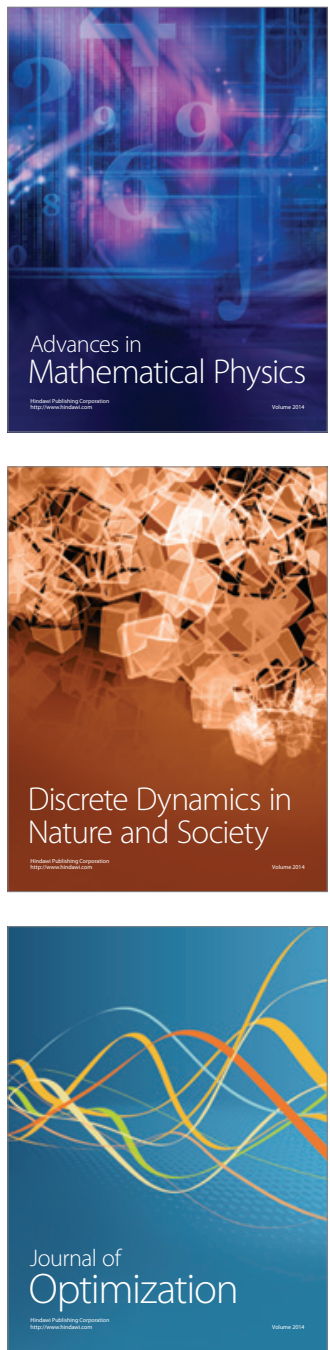